\title{
Bloqueos Regionales en Cirugía Pediátrica Ambulatoria.
}

\author{
Regional Blocks in Pediatric outpatient Surgery \\ https://doi.org/10.25237/carsac2020-10
}

Dr. Ernesto Bermúdez B. ${ }^{1}$, Dra. María Francisca Bernucci P. ${ }^{2}$, Dra. Loreley Bermúdez G.(Ilustraciones). ${ }^{3}$

${ }^{1}$ Servicio de Anestesiología y Reanimación. Clínica Alemana de Santiago. Universidad del Desarrollo.

2 Hospital de Carabineros. Servicio de Anestesiología y Reanimación. Clínica Alemana de Santiago. Universidad del Desarrollo.

${ }^{3}$ Médico EDF CGR Juan Fernández.

Autor Correspopnsal:

Dr. Ernesto Bermúdez B.

Servicio de Anestesiología y Reanimación. Clínica Alemana de Santiago. Universidad del Desarrollo.

Dirección: Av. Vitacura 5951, Santiago, 7650568 Chile.

Teléfono : 56222101699

e-mail : ernbermu@gmail.com

ORCID ID: https://orcid.org/0000-0002-9814-3776

Palabras claves: Bloqueos Regionales, Nervio Periférico, Cirugía Pediátrica Ambulatoria.

Key words: Regional Blocks, Peripheral Nerve, Pediatric Outpatient Surgery.

\section{Resumen}

Introducción: Un porcentaje importante de la cirugía pediátrica se realiza de manera ambulatoria. Esto probablemente se verá acrecentado en el contexto de la pandemia del SARS-Cov2. El uso de la anestesia regional también ha aumentado en la práctica diaria de la anestesia pediátrica.

Objetivo: Resumir el uso de la anestesia regional en la cirugía ambulatoria pediátrica.

Materiales y métodos: Revisión bibliográfica exhaustiva, no sistemática.

Resultados: Existe información de grandes series respecto de los beneficios y seguridad en el uso de anestesia regional en pediatría. El efecto de los bloqueos puede ser prolongado a través del uso de coadyuvantes y bloqueos continuos. Se han descrito y estudiado bloqueos para cabeza y cuello, tronco y extremidades, además de su uso en cirugías específicas.

Conclusión: La cirugía ambulatoria ofrece múltiples beneficios para los pacientes pediátricos. Si bien se han estudiado los principales bloqueos para las cirugías más frecuentes, está pendiente ampliar conocimientos con cohortes más grandes, en el contexto de otras cirugías, nuevos bloqueos y bloqueos continuos, seguridad y protocolos de manejo clínico. 


\begin{abstract}
Introduction: Already a high rate of pediatric surgeries are carried out as ambulatory procedures. This could increase in the context of the SARS-Cov2 pandemia. The use of regional blocks has also increased in the every-day practice of pediatric anesthesia.
\end{abstract}

Objective: To review the knowledge available in the literature on the use of regional anesthesia in the pediatric ambulatory surgery setting.

Materials and methods: A comprehensive, non-systematic review of the literature.

Results: In the last decades, large series have collected data on the benefits and safety regarding the use of regional blocks in pediatric anesthesia. The use of coadyuvants and catheters can help extend the effect of these blocks. We describe and discuss the available evidence on head and neck blocks, truncal and limb blocks and their use for specific ambulatory surgeries.

Conclusion: Ambulatory surgery offers multiple benefits to pediatric patients. The main regional blocks have been studied for the most frequent types of ambulatory surgical procedures. Yet more studies concerning larger cohorts, new and continuous blocks, safety, clinical protocols, and other surgeries, are pending,

\title{
Introducción
}

En la población pediátrica, la cirugía ambulatoria corresponde a un porcentaje importante de las intervenciones en este grupo etario. Esta modalidad permite evitar a los niños problemas inherentes a la hospitalización, como exposición a gérmenes intrahospitalarios, quiebre de rutinas y separación de los padres (1). En el presente año, esto cobra aún más importancia, considerando que en el contexto de la pandemia por el virus SARS-CoV2 se recomienda priorizar las cirugías ambulatorias, dentro de procedimientos electivos necesarios de realizar (2). La anestesia regional (AR) en este contexto aporta con óptimo manejo del dolor, escasos efectos secundarios relevantes y seguridad, lo que permite un alta domiciliaria durante el mismo día. Es por lo tanto el complemento perfecto para la cirugía ambulatoria, cumpliendo con las exigencias y metas de ésta.

En las últimas décadas, la AR ha llegado a ser una piedra angular de la anestesia pediátrica y, en la actualidad, es una técnica usada en la actividad diaria en gran parte de los centros pediátricos. Su uso ha aumentado en forma dramática los últimos años, y en parte esto se debe a la utilización del ultrasonido como guía.

\section{Beneficios y Seguridad de la Anestesia Regional}

Existe una fuerte evidencia en la literatura que el uso del ultrasonido en los bloqueos periféricos disminuye el tiempo de ejecución del bloqueo comparado con electro estimulación, permite utilizar una menor dosis de anestésico local, aumenta el éxito y calidad del bloqueo, medido por consumo de analgésico, duración del bloqueo e intensidad de dolor (3).

En los bloqueos neuroaxiales, el ultrasonido mejora el tiempo de punción, permite predecir la profundidad del espacio peridural, permite visualizar los catéteres y la distribución del anestésico local, y mejora la calidad del bloqueo (3).

En AR pediátrica, uno de los conceptos más importantes es la seguridad. A diferencia de los pacientes adultos, en niños la mayoría de estos procedimientos se realiza bajo anestesia general o sedación. El año 1996 la Asociación Anestesiólogos Francófonos publicó un estudio que incluyó 85.412 pacientes. La AR fue realizada bajo anestesia general en el $89 \%$ de los pacientes, bajo sedación en un $6 \%$ y despiertos un 5\%. Los bloqueos centrales representaron el $61.5 \%$ de todas las técnicas regionales, y los bloqueos de nervio periférico el $31 \%$. La anestesia caudal fue la técnica más usada ( $80 \%$ de bloqueos centrales y $50 \%$ de todas las técnicas regionales). La tasa de complicaciones fue muy baja, $0.09 \%$, todas en bloqueos centrales, y ninguna resultó con secuelas a largo plazo (4). El año 2010 se repite el estudio con135.744 pacientes. La AR fue realizada bajo anestesia general en el $96 \%$ de los casos. En contraste con el reporte anterior, los bloqueos centrales solo representaron el 34\% de las técnicas regionales, y el uso de bloqueos periféricos aumentó a un $66 \%$. Nuevamente, la tasa global de complicaciones fue muy baja, $0.12 \%$, pero fue 6 veces 
mayor en los bloqueos centrales. Ninguna complicación resultó en secuelas a largo plazo (5). Cabe destacar la clara transición de bloqueos centrales a bloqueos periféricos. Esto podría explicarse por la menor morbilidad de los últimos, por la aparición del ultrasonido como guía, y por el mayor entrenamiento y experiencia en bloqueos regionales de los anestesiólogos pediátricos.

Al existir la necesidad de estandarizar procedimientos, resolver controversias y respaldar con evidencia la práctica clínica, las sociedades pediátricas de Europa y Norteamérica crean un comité asesor en temas controversiales en AR pediátrica, donde se dan recomendaciones basadas en la evidencia o en base a la opinión de expertos. Tras el análisis de trabajos a gran escala, se concluye que realizar AR bajo anestesia general en pediatría es tan seguro como hacerlo en pacientes sedados o despiertos, y debiera ser considerado como el estándar de cuidado en pediatría (6). Las complicaciones, como síntomas neurológicos post operatorios $(0.002 \%)$ y toxicidad por anestésicos locales $(0.009 \%)$, son raras y concuerdan con otros reportes en niños y adultos (6-8).

Otro punto importante en relación a la seguridad de la AR en pediatría es la dosis de anestésico local (AL) utilizado. Esto debido a que en diferentes estudios se ha encontrado una amplia variabilidad, siendo en varios casos una masa de droga potencialmente tóxica. En uno de los trabajos que incluyen 40.121 bloqueos, la diferencia de las dosis fue de 5 a 10 veces, dependiendo el tipo de bloqueo (9). En otro trabajo que analiza 18.650 anestesias caudales, el $25 \%$ de los pacientes recibieron una dosis potencialmente tóxica (10).

Las características fisiológicas de los niños los exponen a un mayor riesgo de intoxicación sistémica por anestésicos locales. Los AL son metabolizados por el sistema de citocromos P450 (CyP), CyP3A4 para lidocaína y bupivacaína, y CyP1A2 para ropivacaína. CyP3A4 no está maduro al nacimiento, pero es parcialmente reemplazado por CyP3A7. Al mes de edad el aclaramiento de bupivacaína es $1 / 3$ del adulto y $2 / 3$ a los 6 meses. CyP1A2 no está totalmente maduro antes de los 3 años. El aclaramiento de ropivacaína no alcanza el máximo hasta los 8 años.

Por otro lado, el volumen de distribución está aumentado en recién nacidos y lactantes menores, lo que disminuye las concentraciones pico post inyección única. Pero el riesgo de acumulación post infusión o dosis repetidas está aumentado en relación a los adultos.

La alfa 1 glicoproteína ácida, es la principal proteína a la que los AL se unen. Su concentración es muy baja al nacimiento y aumenta progresivamente durante el primer año de vida. Por lo tanto, en lactantes menores, la fracción libre de anestésico local es mayor. El elevado gasto cardiaco en los niños pequeños acelera la absorción vascular desde los tejidos, produciendo mayores concentraciones iniciales y además disminuyendo la duración de acción. Por lo tanto, las dosis de AL deben disminuirse en menores de 2 años, especialmente al usar infusiones o bolos repetidos. En menores de 1 año el riesgo es mayor, y aún más en menores de 6 meses, en quienes además el hígado es inmaduro.

El año 2018, las sociedades Europeas y de Norteamérica publican las primeras recomendaciones basadas en evidencia, sobre dosis de anestésico local y coadyuvantes (11). Las actuales recomendaciones de dosis de anestésicos locales son las detalladas en las tablas 1 a 4.

Tabla 1: Dosis de anestésico local recomendadas para Anestesia Raquídea (11).

$\begin{array}{llll} & <\text { de } 5 \mathrm{~kg} & 5-15 \mathrm{~kg} & >\text { de } 15 \mathrm{~kg} \\ \text { Bupivacaína hiperbara } & 1 \mathrm{mg} / \mathrm{kg} & 0.4 \mathrm{mg} / \mathrm{kg} & 0.3 \mathrm{mg} / \mathrm{kg}\end{array}$

Tabla 2: Dosis de anestésico local recomendadas para Anestesia Peridural (11).

$\begin{array}{lll} & \text { Caudal } & \text { Lumbar/Torácica } \\ \text { Ropivacaína } 0.2 \% & \text { no superar } 2 \mathrm{mg} / \mathrm{kg} & \text { no superar } 1.7 \mathrm{mg} / \mathrm{kg} \\ \text { Bupivacaína/Levobupivacaína } 0.25 \% & \text { no superar } 2.5 \mathrm{mg} / \mathrm{kg} & \text { no superar } 1.7 \mathrm{mg} / \mathrm{kg}\end{array}$

Tabla 3: Dosis recomendada de Infusión Peridural Continua de Anestésico Local (11) 


$\begin{array}{lll} & \text { Bupi/Levobupivacaína } & \text { Ropivacaína } \\ <3 \text { meses } & 0.0625-0.125 \% 0.2 \mathrm{mg} / \mathrm{kg} / \mathrm{h} & 0.1-0.2 \% 0.2 \mathrm{mg} / \mathrm{kg} / \mathrm{h} \\ <1 \text { año } & 0.125 \% 0.3 \mathrm{mg} / \mathrm{kg} / \mathrm{h} & 0.1-0.2 \% 0.3 \mathrm{mg} / \mathrm{kg} / \mathrm{h} \\ >1 \text { año } & 0.125 \% 0.4 \mathrm{mg} / \mathrm{kg} / \mathrm{h} & 0.1-0.2 \% 0.4 \mathrm{mg} / \mathrm{kg} / \mathrm{h}\end{array}$

Tabla 4: Dosis de anestésico local recomendadas para bloqueos de nervio periférico (11)

Bloqueo Periféricos
Extremidad Superior
Extremidad Inferior
Bloqueos Fasciales
Infusión continúa

Bloqueo Periféricos

continúa

\author{
Ropi/Bupi/Levobupivacaína \\ $0.5-1.5 \mathrm{mg} / \mathrm{kg}$ \\ $0.5-1.5 \mathrm{mg} / \mathrm{kg}$ \\ $0.25-0.75 \mathrm{mg} / \mathrm{kg}$
}

A.L. $0.1 \% 0.1-0.3 \mathrm{mg} / \mathrm{kg} / \mathrm{h}$

Tabla 5: Indicaciones de catéteres de nervio periférico (17)

\begin{tabular}{|c|c|c|}
\hline Localización catéter & Área quirúrgica & Indicación quirúrgica \\
\hline Femoral & Rodilla-Muslo & $\begin{array}{l}\text { LCA }^{*} \text {, fractura Espina tibial, reparación } \\
\text { menisco, }\end{array}$ \\
\hline Canal Aductor & Rodilla & Artroscopía rodilla \\
\hline Ciático-poplíteo & Pierna-Pie & $\begin{array}{l}\text { Osteotomía calcáneo, medio } \\
\text { pie, OTS }{ }^{* *} \text {, fijación externa para } \\
\text { alargamiento }\end{array}$ \\
\hline Plexo Lumbar & Cadera-Muslo & $\begin{array}{l}\text { Reparación labrum, resección tumor } \\
\text { fémur-muslo }\end{array}$ \\
\hline Interescalénico & Hombro-Húmero proximal & $\begin{array}{l}\text { Artroscopía hombro, ,cirugía de húmero } \\
\text { proximal }\end{array}$ \\
\hline Infraclavicular & Codo-Antebrazo-Mano & $\begin{array}{l}\text { OTS húmero distal, } \\
\text { OTS radio-cúbito, } \\
\text { Cirugía codo, polidactilia }\end{array}$ \\
\hline Paravertebral & Tórax & Toracotomía, procedimiento Nuss \\
\hline
\end{tabular}

\section{Prolongación del Bloqueo y Analgesia}

\section{Coadyuvantes}

Existen varios coadyuvantes mencionados en la literatura. Algunos no se recomiendan ya que sus efectos colaterales sobrepasan a los beneficios. Ejemplo de lo anterior son midazolam, neostigmina y buprenorfina. Por otro lado, el uso de epinefrina como coadyuvante no cuenta con estudios en el contexto de cirugía pediátrica ambulatoria. No hay pub- 
licaciones de uso para disminuir la concentración máxima alcanzada en plasma en pacientes ambulatorios y su uso como dosis de prueba para detectar inyecciones intravasculares se recomienda que sea a discreción del operador (6).

La Ketamina es una droga efectiva como coadyuvante, pero actualmente no se recomienda porque la toxicidad demostrada en estudios animales aconseja evitar su uso en recién nacidos y lactantes, por el riesgo de apoptosis neuronal (11).

En la línea de los alfa-2-agonistas, tanto clonidina como dexmedetomidina han demostrado su utilidad. Los estudios evidencian que pueden mejorar el bloqueo de nervio periférico por interferir con las corrientes catiónicas de hiperpolarización (Ih-currents), prolongando la duración del bloqueo y disminuyendo la necesidad de analgesia suplementaria. El efecto de los alfa-2-agonistas parece ser más pronunciado en las fibras C (dolor), que en las fibras A-alfa (motor) (12-13). Los alfa-2-agonistas pueden además producir analgesia por actuar a nivel del cerebro y tronco encefálico, médula espinal y tejidos periféricos.

En relación a la anestesia caudal, esta es una de las técnicas de AR más populares, pero su inconveniente es la corta duración de acción, de 4 a 8 horas. Clonidina y dexmedetomidina por vía caudal prolongan el efecto de los AL. La administración de $1-2 \mathrm{mcg} / \mathrm{kg}$ de dexmedetomidina puede extender significativamente la duración de la analgesia sin aumentar los efectos adversos. La dosis de $1 \mathrm{mcg} / \mathrm{kg}$ es la que tendría mejor relación benefício/riesgo (15). Además, dexmedetomidina tiene la ventaja de disminuir la incidencia de agitación post despertar de anestesia con sevofluorano (14-15).

En un estudio, $1 \mathrm{mcg} / \mathrm{kg}$ de dexmedetomidina administrada por vía caudal o intravenosa, prolonga la duración de la anestesia caudal con similar efectividad (16). Sin embargo, se necesitan más estudios en pediatría para tener una evidencia sólida en relación a cuál es la mejor vía de administración.

Considerando que el paciente pediátrico tiene más riesgo de toxicidad por anestésicos locales comparado al adulto; que las recomendaciones actuales han disminuido las dosis totales de anestésico local, sobre todo en los bloqueos fasciales; que en cirugía ambulatoria adquiere gran importancia la duración del bloqueo regional; y que la dexmedetomidina ha demostrado disminuir la agitación post sevofluorano, es que el uso de coadyuvantes aparece como una alternativa lógica y deseable en esta población de pacientes.

\section{Bloqueos de nervio periférico continuos en cirugía pediátrica ambulatoria.}

Una de los mayores inconvenientes de los bloqueos regionales únicos, aún con la utilización de coadyuvantes, es su limitada duración, por lo que no son ampliamente usados en la cirugía ortopédica mayor. Una solución para brindar alivio del dolor de mayor duración y disminuir la estadía hospitalaria, es a través del uso de los bloqueos continuos de nervio periférico. Aunque su empleo es frecuente en el paciente hospitalizado, el uso en cirugía ambulatoria es muy limitado en la población pediátrica.

Los catéteres de nervio periférico están indicados para procedimientos en los cuales el dolor se extiende por un tiempo mayor a la duración de un bloqueo único, constituyendo un método efectivo para prolongar la analgesia por 48-72 horas post operatorias. Otros beneficios incluyen el alta el día de la cirugía, reducción del consumo de opioides, e incrementar la satisfacción del paciente y la familia (17).

Tabla 5: Indicaciones de catéteres de nervio periférico

En una revisión de más de 2000 catéteres, el porcentaje de complicaciones fue de 12\%. Las complicaciones más frecuentes fueron: desplazamiento, oclusión, falla del bloqueo, infección relacionado al catéter (pacientes que usaron el catéter un promedio de 4.5 días) y punción vascular. No se reportaron complicaciones severas (18).

Uno de los problemas de manejo de los catéteres de nervio periférico es la filtración de AL alrededor del sitio de inserción, lo que puede llevar a la complicación más frecuente en la población pediátrica que es el desplazamiento del catéter. Una estrategia para disminuir la filtración y, por ende, el desplazamiento, es aplicar adhesivo del tipo cianocrilato (19-22) Otra técnica sugerida en la literatura es usar el sistema de catéter sobre la aguja, lo que disminuye la incidencia de filtración (23-24). 
En Chile existen varios centros donde se ha implementado con éxito la analgesia periférica continua en la cirugía en adultos. En pediatría en cambio aún es un área pendiente de desarrollar. Su puesta en marcha mejoraría las condiciones postoperatorias en la población pediátrica y abriría la posibilidad de realizar cirugías más complejas de forma ambulatoria.

\section{Bloqueos}

A continuación, describiremos los bloqueos periféricos más utilizados en la cirugía pediátrica ambulatoria, así como también los más emergentes.

\section{Bloqueos de Cabeza y Cuello.}

La inervación sensorial de la cabeza y cuello está dada principalmente por las 3 ramas principales del nervio trigémino; oftálmico, maxilar y mandibular, junto con las raíces $\mathrm{C} 2-\mathrm{C} 4$, que inervan el cuello y la porción occipital del cuello cabelludo (25-26). La tabla 6 describe los bloqueos de cabeza y cuello utilizados, así como sus indicaciones $\mathrm{y}$ dosis.

Tabla 6: Bloqueos de cabeza y cuello

\section{Bloqueos de tronco para cirugía abdominal.}

El uso del ultrasonido ha permitido el mayor uso de los bloqueos de tronco en cirugías pediátricas abdominales. Este tipo de cirugías incluyen a varios de los procedimientos ambulatorios habituales; hernioplastía, circuncisión, orquidopexia, entre otros. Históricamente, el bloqueo caudal fue la técnica regional de elección en este tipo de cirugías. Es por ello que en la literatura es comparado con cada nuevo bloqueo que se desarrolla. Estas nuevas alternativas aportan flexibilidad al trabajo del anestesiólogo, pudiendo elegir el bloqueo que mejor se adapta a cada caso.

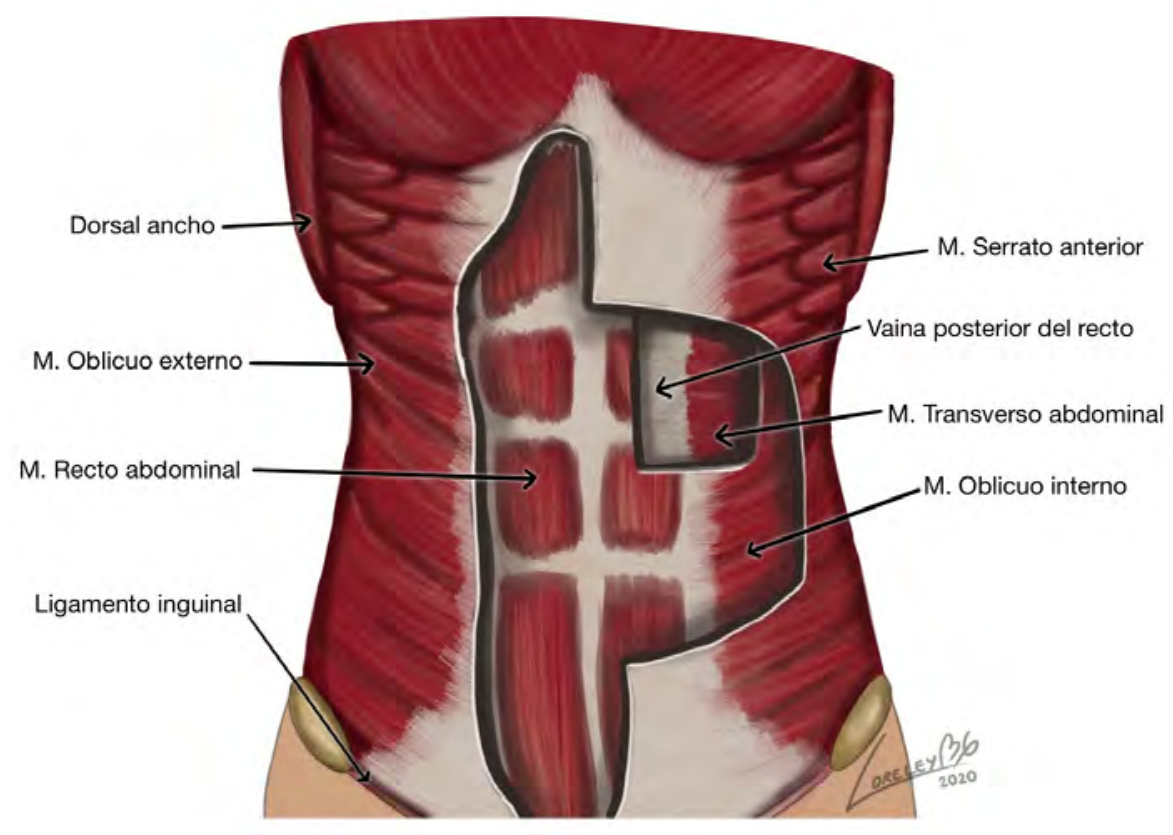

Figura 1. Visualización de musculatura de la pared abdominal.

Tabla 7: Bloqueos de tronco 


\section{Bloqueo de la vaina posterior del recto.}

Este bloqueo se realiza bajo ultrasonido, ubicando el transductor lateral al ombligo. Se inyectan $0.1-0.2 \mathrm{ml} / \mathrm{kg}$ de anestésico local.

Para hernias umbilicales, se ha comparado con el bloqueo caudal e infiltración local. Las tres técnicas otorgaron una analgesia adecuada similar y no mostraron diferencias en cuanto a requerimientos de analgesia ni tiempo para el alta (27). Por lo tanto, la elección de una sobre otro dependerá de las características particulares del paciente, anestesiólogo tratante y recursos del centro.

\section{Bloqueo Ilio-Inguinal-Ilio-Hipogástrico (II/IH).}

En un estudio de pacientes sometidos a cirugía ambulatoria de la región inguinal, se comparó el bloqueo II/IH bajo US, bloqueo II/IH bajo referencias anatómicas y grupo control con analgesia endovenosa sin bloqueo regional. El uso de bloqueo II/IH bajo US mejoró el efecto analgésico intraoperatorio, disminuyó la dosis requerida de AL, y se asoció a una recuperación post operatoria más rápida,(28).

En un estudio de pacientes sometidos a hernioplastia inguinal, el uso del bloqueo II/IH brindó una analgesia similar al uso sólo de paracetamol y no disminuyó delirium postoperatorio, pese a disminuir la dosis total de sevofluorano intra-operatorio. Sin embargo los autores reconocen que el número de pacientes fue calculado para evaluar el efecto del bloqueo II/IH en el delirio post operatorio, y que el resultado en la reducción del dolor post operatorio pudo ser el acotado número de pacientes (29).

\section{Bloqueo del Plano transverso Abdominal, (TAP block).}

El plano transverso abdominal se localiza entre el músculo oblicuo interno y el músculo transverso. Dentro de este plano se encuentran las ramas de los nervios toraco-lumbares que dan inervación sensorial a la pared abdominal anterior. Ubicado el plano bajo ultrasonido, se inyectan $0.2-0.3 \mathrm{ml} / \mathrm{kg}$ de anestésico local.

La evidencia muestra que el TAP block es una técnica efectiva dentro de la analgesia multimodal postoperatoria. Hay que considerar que la distribución de la analgesia a nivel de pared abdominal es limitada, por lo que se debe evaluar el tipo de TAP block a usar para cirugías específicas (lateral, subcostal) (30). También existe evidencia de que el TAP block es una técnica muy segura, con una incidencia de complicaciones de $0.3 \%$, ninguna clínicamente significativa (31).

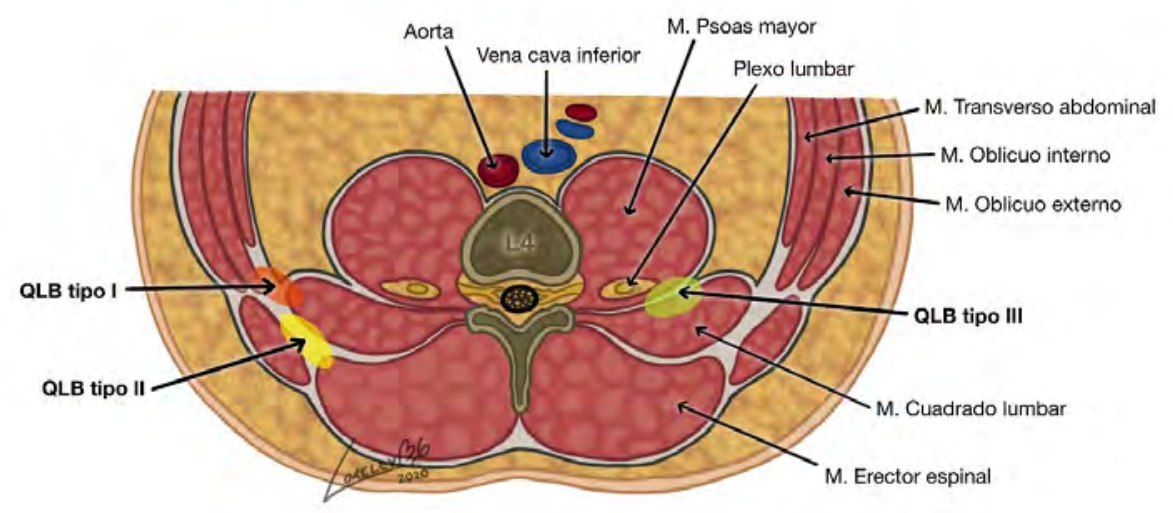

Figura 2. Tipos de Bloqueo Cuadrado Lumbar

\section{Bloqueo Cuadrado Lumbar(QLB)}


El cuadrado lumbar es un músculo de la pared abdominal posterior. Está rodeado por la fascia toraco-lumbar. Existen tres principales abordajes para el QLB. La distribución del anestésico local sería diferente según el abordaje, pero aún no está claramente definido en pediatría. En el abordaje lateral el anestésico local se localiza lateral al cuadrado lumbar y posterior a la fascia toracolumbar. En el abordaje anterior el anestésico local se deposita entre el cuadrado lumbar y el psoas con distribución al espacio paravertebral. En el abordaje posterior se deposita el anestésico local posterior al cuadrado lumbar y lateral o anterior al erector espinal. El anestésico local inyectado rodeando el cuadrado lumbar y posterior a la fascia transversalis puede distribuirse al espacio paravertebral, a lo largo de la fascia endotorácica, bloqueando los nervios somáticos y la cadena simpática torácica de los niveles torácicos inferiores (32).

Se ha comparado el QLB2 versus el TAP block en cirugía de abdomen inferior (hernia inguinal, orquidopexia). El QLB2 logró analgesia postoperatoria de mayor calidad y más prolongada (33). También se ha comparado el uso de QLB2 versus caudal en cirugías de hernia y orquidopexia. El QLB2 se asoció a menor uso de analgésicos en las primeras 24 horas postoperatorias, menor intensidad de dolor (medido por escala FLACC) y mayor satisfacción de los padres de los pacientes. (34)

En cirugía de reimplante ureteral bilateral a través de incisión abdominal infraumbilical, se comparó QLB2 (ropivacaína $0.2 \% 0.5 \mathrm{ml} / \mathrm{kg}$ ) versus bloqueo caudal (ropivacaína $0.2 \%, 1 \mathrm{ml} / \mathrm{kg}$ más morfina $0.03 \mathrm{mg} / \mathrm{kg}$ ), siendo el primero más efectivo en reducir requerimiento de opioides postoperatorios (35).

En otro estudio se comparó el QLB2 con el bloqueo II/IH, ambos guiados por ultrasonido. El QLB2 produjo una mejor analgesia en base a menor consumo de analgesia suplementaria. No se encontraron diferencias en los niveles de dolor postoperatorio (36).

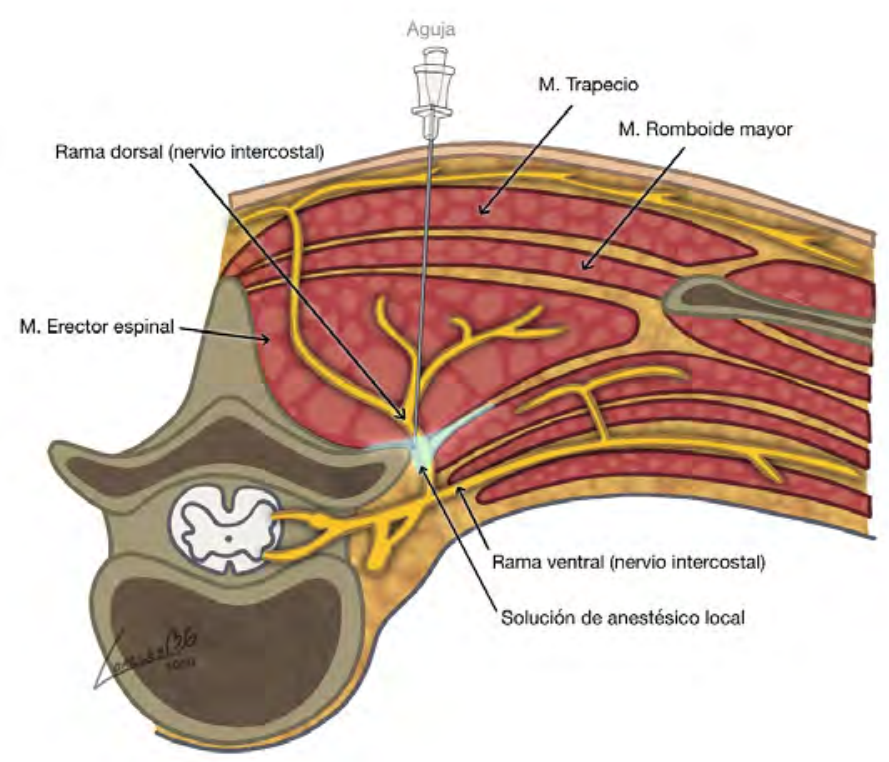

Figura 3. Bloqueo Plano Erector Espinal (ESP)

\section{Bloqueo Plano Erector espinal (ESP).}

Es un bloqueo guiado por ultrasonido que se ha descrito para el manejo del dolor torácico agudo y crónico. La inyección de anestésico local en el plano fascial profundo al músculo erector espinal (ESP), a nivel del proceso transverso de T5 produce analgesia profunda del hemitórax ipsilateral, con distribución cráneo-caudal entre C7 y T8 (37). 
Desde la primera descripción por Forero, diferentes usos de esta técnica se han publicado. En pediatría la mayoría de las publicaciones son reporte de casos y series pequeñas.

En un estudio se comparó los bloqueos II/IH versus ESP, ambos guiados por ultrasonido. El ESP resultó en analgesia más eficiente y de mayor duración que el bloqueo II/IH (38).

En varias revisiones el bloqueo erector espinal ha demostrado ser eficaz en pediatría, pero la mayoría de estas publicaciones son en cirugía pediátrica no ambulatoria.

La llegada del Ultrasonido nos ha permitido mejorar nuestra habilidad para realizar bloqueos periféricos, y así realizar nuevos abordajes que previamente no estaban descritos. Dentro de estos abordajes más recientes tenemos el QLB y el ESP, por lo que sus indicaciones en este grupo etario aún no poseen una evidencia contundente. Se necesitan futuras investigaciones para establecer el rol de cada uno de los bloqueos en la práctica clínica.

\section{Bloqueo Peneano.}

Es un bloqueo muy efectivo en el manejo del dolor postoperatorio de la circuncisión. Existen una variedad de técnicas disponibles, tales como la clásica punción subpúbica, o el bloqueo subcutáneo en anillo. En la punción subpúbica las dosis recomendadas son $0.1-0.2 \mathrm{ml} / \mathrm{kg}$.

Tradicionalmente el bloqueo peneano se realiza en base a referencias anatómicas. Actualmente han aparecido trabajos que comparan esta técnica con el uso del ultrasonido, pero no han mostrado ventajas en relación a la técnica clásica (39-40).

Los diversos estudios que comparan el bloqueo peneano con el bloqueo caudal fueron recopilados en un meta-análisis, que concluyó que ambos bloqueos tienen un efecto analgésico similar. Sin embargo, si bien el bloqueo caudal tendió a tener mayor duración de analgesia, sus efectos no deseados también duraron más, presentando retención urinaria por más tiempo y retardo en la deambulación. En concordancia, los autores sugieren preferir el bloqueo peneano en procedimientos ambulatorios y reservar el bloqueo caudal para pacientes hospitalizados (41).

\section{Bloqueos Extremidad Superior}

La necesidad de utilizar bloqueos de extremidad superior en pediatría es poco frecuente, más aún en el contexto ambulatorio. En el último reporte del PRAN (Pediatric Regional Anesthesia Network) del 2018, de poco más de 100.000 bloqueos regionales realizados entre el 2007 y 2015 , en pacientes pediátricos $0,054 \%$ correspondieron a bloqueos para extremidad superior (42). Las técnicas utilizadas son básicamente las mismas utilizadas en los adultos. Estas se han adaptado a las diferencias que presentan los pacientes pediátricos, siendo una de las más importantes la menor distancia entre estructuras anatómicas.

El bloqueo utilizado con mayor frecuencia fue el supraclavicular, con 2860 casos (42). Por proximidad, puede ocurrir que no haya una clara diferencia clínica final entre este bloqueo y el interescalénico (43). Además, la cercanía con estructuras relevantes como la pleura cervical y la médula espinal cervical obligan también a una punción cuidadosa.

Respecto al bloqueo de nervios terminales, puede existir cierta reticencia a su uso. Lo anterior por temor a lesionar estas finas estructuras, que cuentan con menos tejido conectivo extraneural que el plexo de donde nacen, más aún en niños. Sin embargo, no hay publicaciones al respecto que avalen o desmientan esta preocupación. Los datos aportados por PRAN muestran una tasa global de complicaciones para bloqueos periféricos de 2,8/10 000. Es decir, es difícil obtener datos al respecto ya que son eventos poco frecuentes y además los bloqueos de nervios terminales tienen poca representación en esta base de datos.

Tabla 8: Bloqueos de Extremidad Superior

\section{Bloqueos extremidad inferior}

Los bloqueos de extremidad inferior son los más utilizados como bloqueos únicos tanto como continuos (44). Al igual que como ocurre en los bloqueos de extremidad superior, las técnicas utilizadas derivan de las desarrolladas en adultos, con pequeños ajustes según la anatomía y fisiología del paciente pediátrico. Por ejemplo, al momento de realizar el bloqueo ciático subglúteo, la cara medial del trocánter mayor es hipo ecoica y su tamaño dependerá de la edad del paciente, visualizándose de forma más evidente en niños mayores de 8 años (45). Para el bloqueo ciático 
poplíteo, se ha descrito una amplia variabilidad entre individuos respecto del punto de división del nervio ciático. Esta variabilidad aparece independiente de los factores demográficos. No se han logrado ajustar fórmulas que ayuden a predecir de forma adecuada el punto de división. Con el uso de ultrasonido, Ideo et al observaron en $2 / 3$ de los pacientes la división del ciático se encuentra en el 1/1/4 distal del fémur (45).

En el ámbito de la cirugía ambulatoria pediátrica, los estudios se han centrado principalmente en buscar las mejores estrategias analgésicas para reconstrucción de ligamento cruzado anterior, utilizando bloqueos regionales. Por ejemplo, un estudio comparó tres opciones de bloqueos analgésicos: bloqueo femoral, infiltración intra-articular con anestésico local y la combinación de bloqueo femoral con bloqueo ciático. En el grupo de pacientes con bloqueo femoral asociado a bloqueo ciático, menos del $50 \%$ requirió uso de opioides en el perioperatorio y quienes lo necesitaron, lo hicieron en dosis menores e intensidad del dolor menor (medido con EVA) que los pacientes de los otros grupos (46). Similares resultados se obtuvieron en otro estudio en que todos los pacientes recibían un bloqueo femoral continuo, pero en uno de los grupos se combinó con bloqueo ciático único. En este último grupo, menos pacientes requirieron morfina de rescate para el dolor, en menor dosis total y tuvieron menor dolor máximo (medido por EVA), y una estadía más corta en la Unidad de Recuperación (47). Frente a estos beneficios, deben considerarse también los resultados de un estudio retrospectivo que evaluó el efecto del bloqueo femoral único versus el continuo en la capacidad motora postoperatoria. A los 6 meses post-cirugía, el grupo de pacientes que tuvo un bloqueo continuo presentó asimetría de fuerza entre sus extremidades medida a través de la capacidad de ponerse en cuclillas con una sola pierna a la vez. Aún con esta diferencia, los pacientes de ambos grupos se demoraron una cantidad de tiempo similar para volver a practicar deporte (48).

Tabla 9: Bloqueos de Extremidad Inferior

\section{Conclusión}

La cirugía ambulatoria ofrece múltiples beneficios para los pacientes pediátricos. Esto es aún más cierto en el contexto de la pandemia por SARS-Cov2 que vivimos en la actualidad, en que la modalidad ambulatoria será la de elección para realizar cirugías sensibles al tiempo. La AR aporta con herramientas para cumplir de forma segura con los requerimientos de un peri-operatoria ambulatorio exitoso. Si bien se han estudiado los principales bloqueos para las cirugías más frecuentes, está pendiente ampliar conocimientos con cohortes más grandes, en el contexto de otras cirugías, nuevos bloqueos y bloqueos continuos, seguridad y protocolos de manejo clínico.

\section{REFERENCIAS}

1 A Stein, D Baumgard, I del Rio et al. Updates in Pediatric Regional Anesthesia and Its Role in the Treatment of Acute Pain in the Ambulatory Setting. Curr Pain Headache Rep (2017) 21:11. DOI 10.1007/s11916-017-0614-z

2 Recomendaciones para la cirugía electiva pediátrica segura durante la pandemia COVID-19. Comité de Anestesia Pediátrica SACH - Comité Científico Sociedad Chilena de Cirugía Pediatrica

3 Darren K.M.Lam, BSc, Gareth N. Corry, PhD, and Ban C.H.Tsui, MD. Evidence for the use of Ultrasound Imaging in Pediatric Regional Anesthesia. A Systematic Review. Reg Anesth Pain Med 2015;00: 1-13.

4 Elisabeth Giaufré,MD, Bernard Dalens, MD, and Anne Gombert, MD. Epidemiology and Morbidity of Regional Anesthesia in Children: A one-year Prospective Survey of the French-Language Society of Pediatrics Anesthesiologists. Anesth-Analg 1996;83: 904-12.

5 Claude Ecoffey, MD, Frédéric Lacroix, MD, Elisabeth Giaufré, MD, Gilles Orliaguet, MD, Philippe Courrèges, MD, and asociation des Anesthésistes réanimateurs pédiatriques d'expression Française (Adarpef). Epidemiology and Morbidity of Regional Anesthesia in Children: a follow-up one-year prospective survey of the French-Language Society of Paediatrics Anaesthesiologists (Adarpef). Pediatric Anesthesia 2010; 20: 1061-1069.

6 Giorgio Ivani,MD, Santhanam Suresh, MD, Claude Ecoffey, MD, Adrian Bosenberg, MBChB, FFA(SA), Per- 
Anne Lonnqvist, MD, Elliot Krane, MD, FAAP, Francis Veyckemans, MD, David m. Polaner, MD, FAAP, Marc Van de Velde, MD, and Joseph M. Neal, MD. The European Society of Regional Anaesthesia and Pain Therapy and the American Society of Regional Anesthesia and Pain Medicine Joint Committee Practice Advisory on Controversial Topics in Pediatric Regional Anesthesia. Reg Anesth Pain Med 2015; 40:00-00.

7 N.Llewellyn, RN, RSCN, Ba, and A.Moriarty, FRCA. The National Pediatric Epidural Audit. Pediatric Anesthesia 2007; 17:520-533.

8 Andreas H.Taenzer, MD, MS, Benjamin J.Walker, MD, Adrian T.Bosenberg, MBChB, FFA(SA), Lynn Martin, MD, Santhanam Suresh, MD, David M.Polaner, MD, FAAP, Christie Wolf, MBS, and Elliot J.Krane, MD. Asleep Versus Awake: Does it Matter? Pediatric Regional Block Complications by Patient State: A report Fron thew Pediatric Regional Anesthesia Network. Reg Anesth Pain Med 2014; 39:279-283.

9 S. Suresh and G.S. de Oliveira Jr. Local anaesthetic dosage of peripheral blocks in children: analysis of 40.121 blocks from the Pediatric Regional Anesthesia Network database. s

10 Santhanam Suresh, MD, Justin Long, MD, Patrick K. Birmingham, MD, and Gildasio S. De Oliveira, MD, MSCI. Are Caudal Blocks for Pain Control Safe in Children? An Analysis of 18.650 Caudal Blocks fron the Pediatric Regional Anesthesia Network. Anesth Analg 2015;120:151-6.

11 Santhanam Suresh, MD, Claude Ecoffey, MD, Adrian Bosenberg, MB,ChB, FFA(SA), Per-Anne Lonnqvist, Md, Gildasio S. De Oliveira Jr, MD, MSCi, Oscar de Leon Casasola, MD, José de Andrés, md, PhD, and Giorgio Ivani, MD. The European Society of Regional Anaesthesia and Pain Therapy/American Society of Regional Anesthesia and Pain Medicine Recommendations on Local Anesthetics and Adjuvants Dosage in Pediatric Regional Anesthesia. Reg Anesth Pain Med 2018;43:211-216.

12 Märit Lundblad, Mehdi Trifa, Olfa Kaabachi, Sonia Ben Khalifa, Amjed Fekih Hassen, Thomas Engelhardt, Staffan Eksborg and Per-Arne Lönnqvist. Systematic Review. Alpha-2-adrenoceptor agonists as adjunts to peripheral nerve blocks in children: a meta-analysis. Pediatric Anesthesia 26(2016): 232-238.

13 Märit Lundblad, Daniela Marhofer, Staffan Eksborg and Per-Arne Lönnqvist. Original Article. Dexmedetomidine as adjunt to ilioinguinal/iliohypogastric nerve blocks for pediatric inguinal repair: an exploratory randomized controlled trial. Pediatric Anesthesia 25(2015):897-905.

14 Yao Tong, Hao Ren, Xibing Ding, Shuqing Jin, Zhixia Chen and Quan Li. Analgesic effect and adverse events of Dexmedetomidine as additive for pediatric caudal anesthesia: a meta-analysis. Pediatric Anesthesia 24(2014): 1224-1230.

15 Neerja Bharti,Ranganatha Praveen and Iridu Bala. A dose-response study of caudal dexmedetomidina with ropivacaine in pediatric day care patients undergoing lower abdominal and perineal surgeries: a randomized controlled trial. Pediatric Anesthesia 24(2014): 1158-1163.

16 Yusheng Yao, Chao Yu, Xiaolan Zhang, Yanhua Guo, Xiaochun Zheng. Caudal and intravenous dexmedetomidine similarly prolong the duration of caudal analgesia in children: A randomized controlled trial. Pediatric Anesthesia 2018; 28: 888-896.

17 Christophe Dadure and Xavier Capdevilla. Peripheral catheter techniques. Pediatric Anesthesia 22 (2012) 93-101.

18 B.J.Walker, J.B. Long, G.S.De Oliveira, P. Samuk, C.Setiawan, D.M.Polaner, and S.Suresh, the PRAN investigators. Peripheral nerve catheters in children: An analysis of safety and practice patterns from the pediatric regional anesthesia network(PRAN). British Journal of Anaesthesia ,2015:457-62.

19 Mihaela Visoiu, Lendi N. Joy, Jan S. Grudziak, and Jacques E. Chelly. Original Article. The effectiveness of ambulatory continuous peripheral nerve blocks for postoperative pain management in children and adolescents. Pediatric Anesthesia 24 (2014) 1141-1148.

20 Harshad Gurnaney,MBBS,MPH, F.Wickham Kraemer,MD, Lynne Maxwell, MD, Wallis T. Muhly, MD, Laura Schleelein,MD, and Arjunan Ganesh,MBBS. Ambulatory Continuous Peripheral nerve blocks in children and adolescents: A longitudinal 8-year single center study. Anest-Analg 2014;118:621-7.

21 Andrew Gable, Candice Burrier, Jenna Stevens, Sharon Wrona, Kevin Klingele, Tarun Bhalla, David P Martin, Giorgio Veneziano, Joseph D Tobias. Home peripheral nerve catheters: The first 24 months of experience at a children's hospital. Journal of Pain Research 2016;9:1067-1072.

22 Gurnaney H, Kraemer FW, Ganesh A. Dermabond decreases peri catheter local anesthetic leakage after 
continuous perineural infusions. Anesth Analg 2011;113(1):206

23 B.C.Tsui. Catheter-over-needle method reduces risk of perineural catheter dislocation. British Journal of Anaesthesia 2014;112:759-760.

24 Vivian H.Y.Ip, MBChB. Mark C.Rockley, BScH. Ban C.H.Tsui, MD. The catheter-over-needle assembly offers greater stability and less leakage compared with the traditional counterpart in continuous interscalene nerve blocks: a randomized patient-blinded study. Can J Anesth (2013);60:1272-1273

25 Santhanam Suresh and Polina Voronov. Head and neck blocks in infants, children, and adolescents. Pediatric Anesthesia 22(2012) 81-87

26 Ban C.H.Tsui and Santhanam Suresh, Editors. Pediatric Atlas of Ultrasound-and Nerve Stimulation-Guided Regional Anesthesia 2016

27 . L Relland, J Tobias, D Martin et al. Ultrasound-guided rectus sheath block, caudal analgesia or surgical site infiltration for pediatric umbilical herniorraphy: a prospective, double-blinded, randomized comparison of three regional anesthetic techniques. Journal of Pain Research 2017: 10 2629-2634

28 Lihua Yang, Yucan Xu, Zhongyu Wang, Wei Zhang. Application of Ultrasound-Guided Ilioinguinal/ Iliohypogastric Nerve Blocks in Pediatric Same-Day Surgery. Indian J Surg DOI 10.1007/s12262-015-1301-0

29 Ohashi, S Denda, K Furutani et al Malik, K. and Chamberlain, R.S US ilioinguinal/iliohypogastric block did not reduce emergence delirium after ambulatory pediatric inguinal hernia repair: a prospective randomized doubleblind study. DOI 10.1007/s00595-015-1280-6

30 Christine L.Mai, Mark J.Young and Sadeq A.Quraishi. Review Article. Clinical implications of the transversus abdominis plane block in pediatric anesthesia. Pediatric Anesthesia 22(2012) 831-840

31 Justin B. Long, MD, Patrick K. Birmingham, MD, Gildasio S. De Oliveira, Jr, MD, MSCI, Katie M. Schaldenbrand,MPH, and Santhanam Suresh, MD. Transversus Abdominis Plane Block in Children: A Multicenter Safety Analysis of 1994 Cases from the PRAN(Pediatric Regional Anesthesia Network)Database. Anesth-Analg 2014;119:395-9-

32 Dam M, Moriggl B, Hansen CK, Hoermann R, Bendtsen TF, Børglum J: The pathway of injectate spread with the transmuscular quadratus lumborum block: A cadaver study. Anesth-Analg 2017;125:303-12.

33 Gözen Öksüz,MD, Bora Bilal, MD, Yavuz Gürkan,MD, Aykut Urfalioglu,MD, Mahmut Arslan,MD, Gökçe Gisi,MD, and Hafize Ösküz,MD. Quadratus Lumborum Block Versus Transversus Abdominis Plane Block in Children Undergoing Low Abdominal Surgery. Reg Anesth Pain Med 2017 ;vol 42, No5

34 G Oksuz, M Arslan, A Urfalioglu et al Comparison of quadratus lumborum block and caudal block for postoperative analgesia in pediatric patients undergoing inguinal hernia repair and orchidopexy surgeries: a randomized controlled trial. Reg Anesth Pain Med 2020;0:1-5. doi:10.1136/rapm-2019-101027.

35 Makoto Sato. Ultrasound-guided quadratus lumborum block compared to caudal ropivacaine/morphine in children undergoing surgery for vesicoureteric reflex. Pediatric Anesthesia 2019;00:1-6

36 Artid Samerchua, Prangmalee Leurcharusmee, Kachain Panichpichate, Nutchanart Bunchungmongkol, Mullika Wanvoharn, Kanokkan Tepmalai, Jiraporn Khorana, Sireekarn Chantakhow. A prospective, randomized comparative study between ultrasound-guided posterior quadratus lumborum block and ultrasound guided ilioinguinal/iliohypogastric nerve block por pediatric inguinal herniotomy. Pediatric Anesthesia 2020;30:498-505-

37 Mauricio Forero, MD,FIPP, Sanjib D.Adhikary,MD, Hector Lopez, MD, Calvin Tsui,BMS, and Ki Jinn Chin,MBBS, MMed, FRCPC. The Erector Spinae Plane Block. A Novel Analgesic Technique in Thoracic Neuropathic Pain. Reg Anesth Pain Med vol 41, No 5, 2016.

38 El-Eman,ESM, El motlb EA. Ultrasound-guided erector spinae versus ilioinguinal/iliohypogastric block for postoperative analgesia in children undergoing inguinal surgeries. Anesth Essays Res 2019;13:274-9-

39 Michael J. O”Sullivan, Branislav Mislovic and Elise Alexander. Original Artricle. Dorsal penile block for male pediatric circumcision-randomized comparison of ultrasound -guided vs anatomical landmark technique. Pediatric Anesthesia 21(2011) 1214-1218

40 An Teunkens, Marc Van de Velde, Kristien Vermeulen, Philippe Van Loon, Guy Bogaert, Steffen Fieuws, Steffen Rex. Dorsal penile nerve block for circumcision in pediatric patients: A prospective, observer-blinded, 
randomized controlled clinical trial for the comparison of ultrasound-guided vs landmark technique. Pediatric Anesthesia 2018;28:703-709.

41 K Malik and RS Chamberlain. Penile Blocks Demonstrate Similar Reliability and Efficacy in Pediatric Patients Undergoing Circumcision: A Meta-Analysis. International Journal of Clinical Medicine,7,309-319. http:// dx.doi.org/10.4236/ijcm.2016.75033

42 B Walker, J Long, M Sathyamoorthy et al. Complications in Pediatric Regional Anesthesia. An analysis of More than 100,000 block from the Pediatric Regional Anesthesia Network. Anesthesiology 2018;129:721-32

43 P Marhofer, H Willschke, and S Kettner. Ultrasound-guided upper extremity blocks-tips and tricks to improve the clinical practice. Pediatric Anesthesia 22 (2012) 65-71 doi:10.1111/j.1460-9592.2011.03744.x

44 B Tsui, S Suresh. Ultrasound Imaging for Regional Anesthesia in Infants, Children, and Adolescents. A review of Current Literature and Its Application in the Practice of Extremity and Trunk Blocks. Anesthesiology 2010; 112:473-92.

45 S Ideno, N Miyazawa, A Shinto et al. Distribution of sciatic nerve division in children: a prospective observational study using an ultrasound device. Pediatric Anesthesia 27 (2017) 211-218

46 L Santana, J Lovejoy, G Kiebzak, et al. Comparison of Pain Score and Medication Usage Between 3 Pain Control Strategies for Pediatric Anterior Cruciate Ligament Surgery. Cureus 11(8): e5498. DOI 10.7759/ cureus.5498.

47 A Daoud, T Mandler, A Gagliardi et al. Combined Femoral-Sciatic Nerve Block is Superior to Continuous Femoral Nerve Block during anterior cruciate ligament reconstruction in the pediatric population. The Iowa Orthopedic Journal vol 38, 101-106.

48 H Parikh, A Gagliardi, D Howell et al. Femoral Nerve Catheters are Associated with Limb Strength Asyetry at 6 months after primary ACL Reconstruction in Pediatric Patients. doi: 10.1111/PAN.13983. 